\title{
Influence of the cloud-level neutral layer on the vertical propagation of topographically generated gravity waves on Venus
}

\author{
Takeru Yamada ${ }^{1 *} \mathbb{D}$, Takeshi Imamura ${ }^{2}$, Tetsuya Fukuhara ${ }^{1}$ and Makoto Taguchi ${ }^{1}$
}

\begin{abstract}
The reason for stationary gravity waves at Venus' cloud top to appear mostly at low latitudes in the afternoon is not understood. Since a neutral layer exists in the lower part of the cloud layer, the waves should be affected by the neutral layer before reaching the cloud top. To what extent gravity waves can propagate vertically through the neutral layer has been unclear. To examine the possibility that the variation of the neutral layer thickness is responsible for the dependence of the gravity wave activity on the latitude and the local time, we investigated the sensitivity of the vertical propagation of gravity waves on the neutral layer thickness using a numerical model. The results showed that stationary gravity waves with zonal wavelengths longer than $1000 \mathrm{~km}$ can propagate to the cloud-top level without notable attenuation in the neutral layer with realistic thicknesses of 5-15 km. This suggests that the observed latitudinal and local time variation of the gravity wave activity should be attributed to processes below the cloud. An analytical approach also showed that gravity waves with horizontal wavelengths shorter than tens of kilometers would be strongly attenuated in the neutral layer; such waves should originate in the altitude region above the neutral layer.
\end{abstract}

Keywords: Vertical propagation, Gravity waves, Neutral layer, Venus

\section{Introduction}

A surprising discovery on the Venus atmosphere in the 2010s is a bow-shaped temperature structure by Longwave Infrared Camera (LIR) onboard the Venus orbiter Akatsuki (Fukuhara et al. 2017b). LIR detects thermal infrared radiation from the cloud top at an altitude of approximately $65 \mathrm{~km}$ and provides images of the horizontal distribution of the temperature at this height (Fukuhara et al. 2011, 2017a; Taguchi et al. 2007). The bow-shaped structure extended from the northern hemisphere to the southern hemisphere with a length of approximately 10,000 km (Fukuhara et al. 2017b). The bow-shaped structure remained at the geographical location around the local time of 16:00 for at least 5 days, despite the strong westward wind of about $100 \mathrm{~m}$ $\mathrm{s}^{-1}$ blowing at the cloud-top altitude. The location corresponds to Aphrodite Terra, a large highland. Fukuhara

*Correspondence: takeru.yamada@rikkyo.ac.jp

${ }^{1}$ Graduate School of Science, Rikkyo University, Tokyo 171-8501, Japan

Full list of author information is available at the end of the article et al. (2017b) interprets the bow-shaped structure as an atmospheric gravity wave excited by flows impinging on the topography, e.g. mountain waves. Hereafter, the bowshaped structures are called the stationary gravity waves.

Analyzing the LIR data, Kouyama et al. (2017) reported that stationary gravity waves periodically appeared above four specific mountainous regions with heights of higher than $5 \mathrm{~km}$ and widths of wider than $3 \mathrm{~km}$ in the latitude region within $\pm 30^{\circ}$ when they are in the afternoon. The typical temperature amplitude of the waves is $\sim 0.5 \mathrm{~K}$ at noon, and increases to $\sim 1.0 \mathrm{~K}$ toward the evening and decreases thereafter. Stationary waves are almost undetectable from midnight to the morning.

Navarro et al. (2018) reproduced these local timedependent appearances of stationary waves in a general circulation model by applying a parameterized mountain wave stress to the lower atmosphere. They suggested that the local time dependence of the stationary waves is caused by the accelerated winds near the surface induced by the diurnal cycle of convection in the lower atmosphere. 
A mountain wave is vertically propagating $\left(m^{2}>0\right)$ when $\omega^{*}<N$, while it is evanescent $\left(m^{2} \leq 0\right)$ when $\omega^{*}>N$, where $m$ is the vertical wavenumber, $\omega^{*}=|k u|$ is the positive intrinsic frequency with $k$ being the zonal wavenumber and $u$ being the background zonal wind velocity, and $N$ is the buoyancy frequency. The waves should be evanescent in a neutral layer where $N=0$. The energy density of an evanescent wave decreases exponentially with altitude, e.g. Salby (1996). Therefore, mountain waves are thought to be attenuated in a neutral layer.

There is a neutral layer at the altitude range of 50 to $60 \mathrm{~km}$ in the Venus atmosphere due to heating of the cloud base at altitude of $\sim 50 \mathrm{~km}$ by upwelling infrared radiation (Seiff et al. 1985). To what extent gravity waves can propagate vertically through the neutral layer has been unclear. The thickness of the neutral layer seems to change with local time; radio occultation measurements by Akatsuki showed that the thickness of the neutral layer at low latitudes is variable and up to $10 \mathrm{~km}$ from midnight to the morning and that it is $4-5 \mathrm{~km}$ in the afternoon (Imamura et al. 2017). Such a local time dependence has been predicted by a mesoscale model (Imamura et al. 2014): cloud-level convection should be enhanced on the nightside where net radiative cooling of the cloud top is stronger. Stationary gravity waves have been observed extensively in the afternoon region where the neutral layer is relatively thin. Hence, we assume that the local time dependence of the neutral layer thickness is responsible for the periodic appearance of the stationary gravity waves. Furthermore, the thickness of the neutral layer also varies with latitude in such a way that it is greater at high latitudes, according to Venus Express radio occultation (Tellmann et al. 2009); the disappearance of stationary waves at high latitudes might be attributed to the thickening of the neutral layer.

To date, the characteristics of the gravity waves propagating in the neutral layer in the Venus atmosphere has not been studied intensively. Young et al. (1987, 1994) documented the propagation characteristics of topographic gravity waves with the zonal wavelength of 400 $\mathrm{km}$ associated with altitude variations of the zonal wind and the stability of the background state. However, these studies have not examined the influence of thickening the neutral layer on the vertical propagation of the gravity waves. In the present study, we clarify the vertical propagation characteristics of the gravity waves in the Venus atmosphere with different neutral layer thicknesses using a numerical model.

\section{Numerical model}

We used a numerical model developed by Imamura (2006) and Fukuhara et al. (2017b). The model solves primitive equations in spherical geometry that are finite differenced with a staggered vertical and horizontal grid system (grid C) as described by Haltiner and Williams (1980). The horizontal velocity and the temperature are integrated in time using a centered flux-form space difference, Euler backward (Matsuno) scheme, and the vertical velocity in the pressure coordinate and the geopotential are diagnosed with boundary conditions at each time step. The model covers the altitude region from $5 \mathrm{~km}\left(6.665 \times 10^{6} \mathrm{~Pa}\right)$ to approximately $88 \mathrm{~km}(6.665 \mathrm{~Pa})$, which is divided into 100 levels at a regular spacing in log-pressure altitude. The horizontal grid interval is $1^{\circ}$ both in longitude and latitude. Time integration starts from a basic state and is continued until reaching an equilibrium state with a time step of $5 \mathrm{~s}$. Since the temporal development of the background state is beyond the scope of this study, the velocity and the temperature fields are divided into their zonal-mean and disturbance, and the former is set to the initial value at each time step.

In our model the atmosphere rotates from east to west and westward winds are taken to be positive. The basic zonal velocity profile $\bar{u}_{\text {eq }}$ on the equator is given by the polynomial function adopted in Schubert and Walterscheid (1984); the velocity is $1.3 \mathrm{~m} \mathrm{~s}^{-1}$ at $5 \mathrm{~km}$, increasing monotonically with height to $94 \mathrm{~m} \mathrm{~s}^{-1}$ at $68 \mathrm{~km}$. The velocity above the cloud top is the average of the empirical model A (negative shear above the cloud top) and the model B (no shear above the cloud top) given in the paper above. The basic temperature profile on the equator was calculated from the buoyancy frequency $N$ as

$$
\frac{\partial T(\sigma)}{\partial \sigma}=\frac{H(\sigma)}{\sigma}\left(\frac{g}{c_{p}}-\frac{T(\sigma)}{g} N(\sigma)^{2}\right) .
$$

Here, $\sigma=p / p_{b}$ is the vertical coordinate, where $p$ is the pressure and $p_{b}$ is the pressure at the lower boundary; $T(\sigma)$ is the temperature; $H(\sigma)=R T(\sigma) / g$ is the scale height, where $R=191.4 \mathrm{~J} \mathrm{~kg}^{-1} \mathrm{~K}^{-1}$ is the gas constant and $g=8.87 \mathrm{~m} \mathrm{~s}^{-2}$ is gravitational acceleration; and $c_{p}=821 \mathrm{~J} \mathrm{~kg}^{-1} \mathrm{~K}^{-1}$ is the specific heat at constant pressure (Seiff et al. 1985). The temperature at the lower boundary of $5 \mathrm{~km}$ altitude, which is used in integrating Eq. (1), is $T(\sigma=1)=696.8 \mathrm{~K}$ based on Venus International Reference Atmosphere (Seiff et al. 1985), and the vertical profile of $N$ is taken from model $\mathrm{A}$ in Schubert and Walterscheid (1984) with the boundary value at the surface $(0 \mathrm{~km})$ being $2.0 \times 10^{-3} \mathrm{~s}^{-1}$.

The equations are integrated in a coordinate system rotating at the angular velocity corresponding to the equatorial velocity at the bottom altitude of $\bar{u}_{\mathrm{eq}}(\sigma=1)=1.3 \mathrm{~m} \mathrm{~s}^{-1}$. The meridional distribution of the zonal velocity in this coordinate system is given by

$$
\bar{u}(\phi, \sigma)=\xi(\phi) \bar{u}_{\mathrm{eq}}(\sigma)-\bar{u}_{\mathrm{eq}}(\sigma=1) \cos \phi .
$$




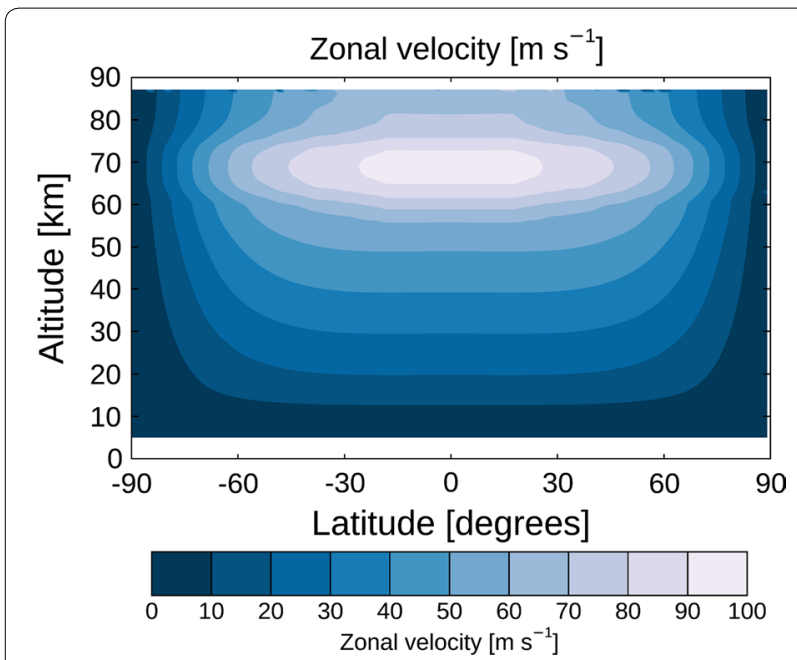

Fig. 1 Meridional distribution of the basic zonal velocity

Here, $\phi$ is the latitude and $\xi$ is defined as follows:

$\xi(\phi)=\left\{\begin{array}{l}0.6\left(1+0.4 \phi^{2}-0.4 \phi^{4}\right)+0.4 \cos \phi\left(|\phi|<75^{\circ}\right) \\ 0.6(2.05 \cos \phi)+0.4 \cos \phi\left(|\phi| \geq 75^{\circ}\right)\end{array}\right.$.

Figure 1 shows the calculated meridional distribution of the basic zonal velocity. The latitudinal profile at the cloud top is basically consistent with measurements by cloud tracking (Horinouchi et al. 2018; Rossow et al. 1990). The meridional distribution of the basic temperature was calculated from the basic zonal velocity using the thermal wind balance in cyclostrophic regime with the equatorial velocity profile $\bar{u}_{\text {eq }}$ as the boundary condition:

$$
\frac{\partial T(\phi, \sigma)}{\partial \phi}=\frac{\sigma a}{R}\left(f \frac{\partial \bar{u}(\phi, \sigma)}{\partial \sigma}+\frac{\tan \phi}{a} \frac{\partial \bar{u}(\phi, \sigma)^{2}}{\partial \sigma}\right) .
$$

Here, $a=6050 \mathrm{~km}$ is the radius of Venus and $f=2 \Omega \sin \phi$ is the Coriolis parameter, where $\Omega=2.15$ $\times 10^{-7} \mathrm{rad} \mathrm{s}^{-1}$ is the angular velocity corresponding to $\bar{u}_{\text {eq }}(\sigma=1)$. The dry convective adjustment was applied to keep the static stability positive or neutral. The basic meridional velocity is zero everywhere.

To investigate the effect of the neutral layer on the propagation of gravity waves, we conduct a numerical experiment with different neutral layer thicknesses in the basic state. Figure 2 shows vertical profiles of the static stability, $S(z)=\frac{\mathrm{d} T(z)}{\mathrm{d} z}+\frac{g}{c_{p}}$ where $z$ is the altitude in unit of kilometers, on the equator for four cases of the neutral layer thickness. The static stability above the neutral layer differs among the experiments. This is because the temperature profile is calculated from Eq. (1) with a common $N^{2}$ profile adopted from Schubert and Walterscheid

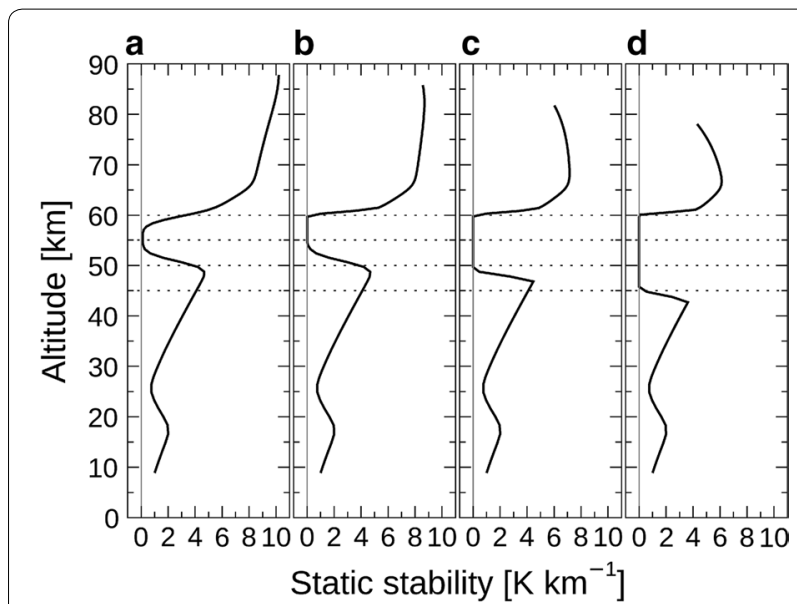

Fig. 2 Vertical profiles of the basic-state static stability on the equator. a Nominal case, b neutral layer thickness of $5 \mathrm{~km}, \mathbf{c} 10 \mathrm{~km}$, and $\mathbf{d} 15 \mathrm{~km}$

(1984) except for the neutral layer. The nominal case was calculated as described above and correspond to a neutral layer thickness of $\sim 2 \mathrm{~km}$. The other three cases represent increased neutral layer thicknesses of 5, 10, and 15 $\mathrm{km}$.

A temperature forcing exciting gravity waves is imposed on the lower boundary with a Gaussian-shaped function $\hat{T} \times \exp \left[-\left(\delta \lambda^{2}+\delta \phi^{2}\right) /\left(b^{2}\right)\right]$, where $\hat{T}=1 \mathrm{~K}$ is the amplitude, $\delta \lambda$ and $\delta \phi$ are the longitudinal and latitudinal distances from the center of the forcing, and $b=3^{\circ}$ is the half-width. The center of the forcing is located at a longitude of $180^{\circ} \mathrm{E}$ on the equator. The full width of the topographic rise contributing to the wave generation will be around half of the horizontal wavelength of the wave; given the horizontal wavelengths of $\sim 1500 \mathrm{~km}$ above Aphrodite Terra as observed by LIR (Fukuhara et al. $2017 \mathrm{~b})$, the half width $b$ is taken to be $3^{\circ}$ corresponding to $\sim 300 \mathrm{~km}$. The amplitude $\hat{T}$ is taken to be $1 \mathrm{~K}$ so that the temperature variation observed at the cloud top is reproduced.

Physical processes introduced in this model are described below. Figure 3 shows the damping rate along the altitude, which is given in unit of $\mathrm{d}^{-1}$ ( $\mathrm{d}$ is the Earth day) as

$$
r=\frac{1}{20 \times \exp [-(z-60) / 10]}+\frac{1}{1500 \times \exp [-(z-60) / 2.8]}
$$

where $z$ is the altitude in unit of kilometers. The first term on the right-hand side represents the radiative relaxation rate based on the radiative transfer modeling by Crisp (1989). The second term represents the attenuation in a sponge layer. The attenuation by the sponge layer dominates the total damping above $\sim 77 \mathrm{~km}$ to prevent wave 


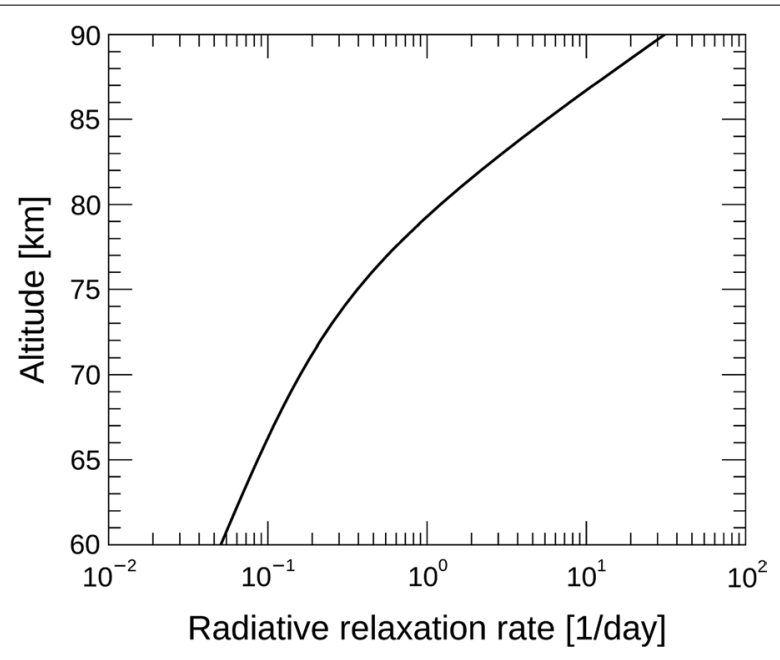

Fig. 3 Radiative relaxation rate as a function of altitude

reflection near the upper boundary by attenuating the disturbance temperature and wind velocity at fast damping rates; for example the relaxation time at $80-90 \mathrm{~km}$ is less than one earth day. The vertical diffusion coefficient is $0.3 \mathrm{~m}^{2} \mathrm{~s}^{-1}$ for all layers; though radio scintillation measurements provided values of $0.2 \mathrm{~m}^{2} \mathrm{~s}^{-1}$ and $4 \mathrm{~m}^{2} \mathrm{~s}^{-1}$ at $45 \mathrm{~km}$ and $60 \mathrm{~km}$, respectively (Woo and Ishimaru 1981; Woo et al. 1982), we focus more on the altitude region below the cloud top. This study neglects the effect of enhanced diffusion due to convection in the neutral layer. Horizontal diffusion is not included in the model. A horizontal diffusion coefficient same as the vertical diffusion would lead to a damping time much longer than the intrinsic period for the wave parameters adopted in the present study. Though large-scale horizontal turbulence may contribute, they are not constrained by observations.

To compare the temperature disturbance calculated by the simulation with the bow-shaped structures observed by LIR, we considered the effect of vertical averaging of LIR sounding. The Full Width Half Maximum (FWHM) of the weighting function of LIR is $10 \mathrm{~km}$ with a center altitude of $65 \mathrm{~km}$ (Taguchi et al. 2007). Hence, the temperature disturbance obtained by the simulation is vertically integrated with the weighting function from 60 to $80 \mathrm{~km}$ as

$$
T_{W}^{\prime}(\lambda, \phi, z)=\int_{60 \mathrm{~km}}^{80 \mathrm{~km}} W(z) T^{\prime}(\lambda, \phi, z) \mathrm{d} z .
$$

Here, $T_{W}^{\prime}(\lambda, \phi, z)$ is the temperature disturbance to be observed, $W(z)$ is the weighting function of LIR (Fig. 6 of Taguchi et al. (2007)), $T^{\prime}(\lambda, \phi, z)$ is the temperature disturbance, and $z$ is the altitude. Hereafter, the temperature disturbance means $T_{W}^{\prime}$. Though the center altitude

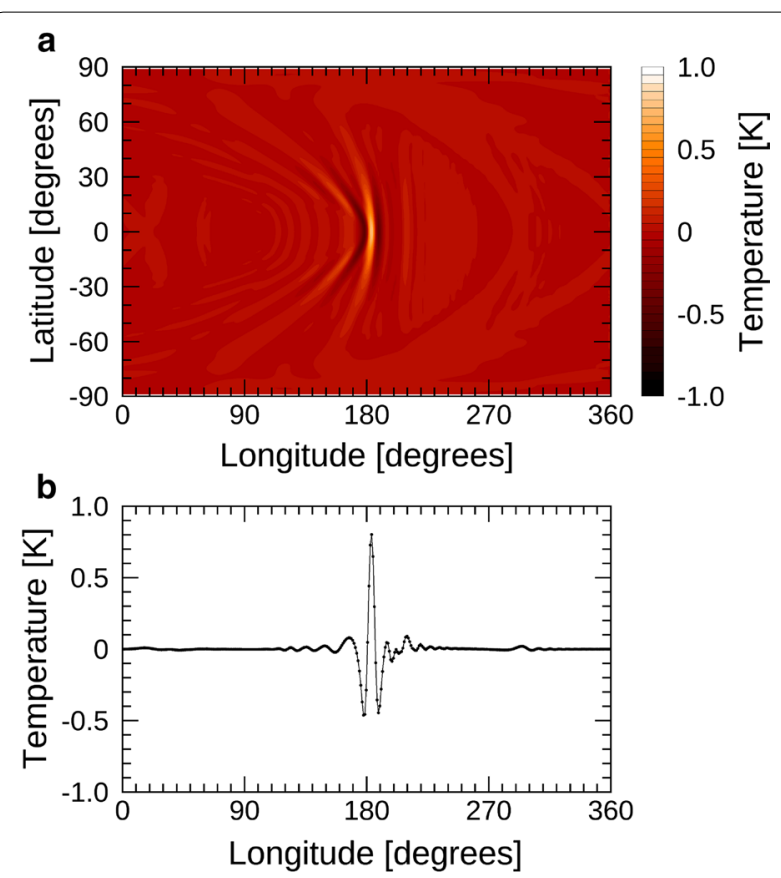

Fig. 4 a Horizontal structure of the temperature disturbance and $\mathbf{b}$ longitudinal profile of the temperature disturbance averaged for the latitudes of $-5^{\circ}$ to $5^{\circ}$ in the nominal case shown in Fig. 2

of the weighting function also varies with wave's phase because of the wave-induced vertical advection of clouds, we assume the center altitude is unchanged; this effect should be considered in future studies.

\section{Results}

The results shown below were obtained by analyzing data on 5th earth day where the temperature disturbance at the cloud top $(65 \mathrm{~km})$ reached a quasi-steady state. Figure 4a shows the horizontal temperature structure. A bow-shaped temperature disturbance is reproduced at the cloud-top level. This structure has an apex on the equator. Both edges of the bow extend to the latitudes beyond $30^{\circ}$. These features resemble the bow-shaped temperature structure observed by LIR (Fukuhara et al. 2017b; Kouyama et al. 2017). Figure 4b shows the longitudinal profile along the equator, indicating that the peak-to-peak amplitude of the temperature disturbance has a maximum value of $\sim 1.33 \mathrm{~K}$ at the longitude shifted eastward by $\sim 5^{\circ}$ from the forcing. From Fig. $4 \mathrm{~b}$, the zonal wavelength is $\sim 12^{\circ}$ on the eastern half of the wave packet and $\sim 18^{\circ}$ on the western half, corresponding to $1300 \mathrm{~km}$ and $1900 \mathrm{~km}$, respectively.

Figure 5a shows the longitude-altitude cross section of the temperature disturbance on the equator. Waves with phase lines titled eastward with height are identified 


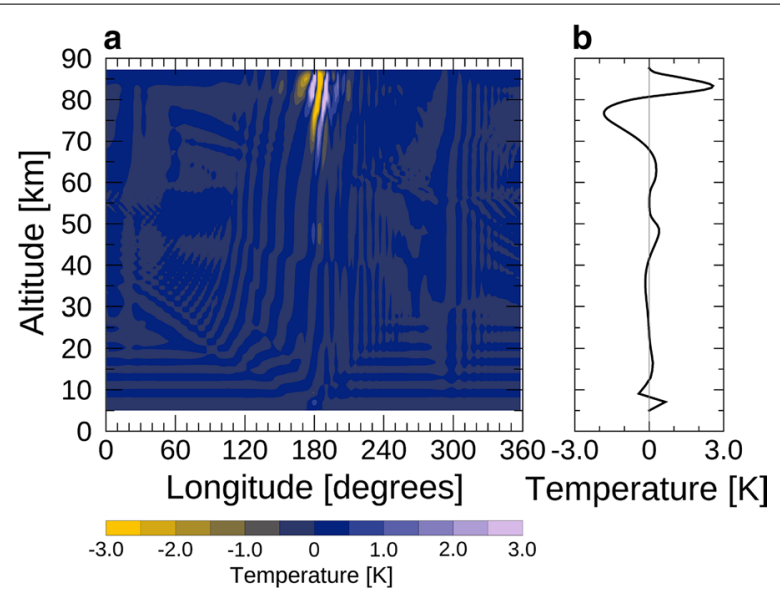

Fig. 5 a Longitude-altitude cross section of the temperature disturbance on the equator for the nominal case shown in Fig. 2. The zonal wind direction is right to left. $\mathbf{b}$ Vertical profile of the temperature disturbance just above the position of the wave forcing. Note that regions of $3 \mathrm{~K}$ and above were filled with purple, and regions of $-3 \mathrm{~K}$ and below were filled with yellow (a)

in the entire altitude region in the longitude from 180 to $210^{\circ} \mathrm{E}$, which includes the forcing region. The waves seem to produce the temperature disturbance above the neutral layer.

The vertical profile of the waves (Fig. 5b) clearly shows an oscillating structure. Regarding the length between adjacent local maxima as the vertical wavelength, the vertical wavelength is estimated to be approximately 30-40 $\mathrm{km}$. The dispersion relation for a mountain wave in a uniform zonal wind and static stability field can be written in the form $m^{2}=N^{2} / \bar{u}^{2}-k^{2}$ (Durran 1990). Since $m \gg k$, we obtain the vertical wavelength as $\lambda_{z}=2 \pi(\bar{u} / N)$. Substituting the background zonal velocity of $90.4 \mathrm{~m} \mathrm{~s}^{-1}$ and the buoyancy frequency of $0.02 \mathrm{~s}^{-1}$ at the cloud top $(65 \mathrm{~km})$ in this model into this relation, we obtain $\lambda_{z} \sim 30 \mathrm{~km}$, which is consistent with the vertical wavelength estimated from Fig. 5b. The vertical wavelength estimated from this relation diverges in the neutral layer, suggesting that the gravity wave becomes evanescent and the phase becomes constant with altitude. This explains the almost vertical phase lines in the altitude of 50-60 $\mathrm{km}$ shown in Fig. 5a. At other altitudes, the vertical wavelength is $15-45 \mathrm{~km}$.

Figure $5 \mathrm{~b}$ shows that the temperature disturbance decreases to nearly zero in the altitude range of 52-56 $\mathrm{km}$, i.e. the near-neutral layer. According to Hinson and Jenkins (1995), the amplitude of the temperature disturbance $\left|T^{\prime}\right|$ associated with a gravity wave is related to the background state as $T^{\prime} \propto \bar{T} \bar{\rho}^{-\frac{1}{2}} N^{\frac{3}{2}}$, where $\bar{T}$ is the background temperature and $\bar{\rho}$ is the background density. This relation explains the small amplitude in the altitude region where $N$ is locally small: the value of $N^{\frac{3}{2}}$ at $52-56$ $\mathrm{km}$ is ten times smaller than those outside this region, while $\bar{T}$ and $\bar{\rho}^{-\frac{1}{2}}$ change smoothly with altitude throughout the model domain except the change of the vertical gradient of $\bar{T}$ by $\sim 8 \%$ around 52 and $56 \mathrm{~km}$ altitude.

Similar bow-shaped structures also appear in the other cases except that the amplitude decreases with increasing the neutral layer thickness as shown in Fig. 6. The maximum peak-to-peak amplitudes for the neutral layer thickness of $5 \mathrm{~km}, 10 \mathrm{~km}$, and $15 \mathrm{~km}$ are $1.21 \mathrm{~K}, 0.95 \mathrm{~K}$, and $0.38 \mathrm{~K}$, respectively. The results imply that the almost complete disappearance of bow-shaped features in the morning and in the high latitudes cannot be explained by the increased thickness of the neutral layer. In addition, it can be seen from Fig. $6 \mathrm{~d}-\mathrm{f}$ that wave ducting occurs below the neutral layer as the neutral layer becomes thicker. As a result, gravity waves propagate horizontally from the excitation source.

\section{Discussion}

It was shown that the stationary gravity waves under consideration are not strongly attenuated in the neutral layer and that the thickening of the neutral layer in the midnight-morning and in the high latitude region cannot explain the disappearance of stationary waves. To understand this result, let us examine the behavior of gravity waves in a neutral layer using a linear, analytical solution. Here, we ignore the rotation of the planet because the intrinsic period of the wave (several hours) is much shorter than the planetary rotation period ( 243 earth days). The vertical shear of the background wind is also neglected because convection in the neutral layer is expected to mix momentum vertically to weaken the vertical shear; though the vertical shear is not small in the neutral layer in the model, the shear does not seem to prevent vertical propagation, and thus the present analysis focusing on the effect of the static stability is considered appropriate. The turbulent diffusion will not severely affect wave propagation; assuming a turbulent diffusion coefficient of $K \sim 200 \mathrm{~m}^{2} \mathrm{~s}^{-1}$ in the convective layer (Imamura and Hashimoto 2001) and a vertical wavelength of $\lambda_{z} \sim 30 \mathrm{~km}$, the damping time is calculated to be $\left(\lambda_{z} / 2 \pi\right)^{2} / K \sim 32$ hours, which is much longer than the wave's intrinsic period. Considering also that the thickness of the convective layer is much less than the vertical wavelength, the effect will be even less.

We assume a wave solution of the form

$$
w(x, z, t) \propto \exp \left[i k x+i m z+i \omega t+\frac{z}{2 H}\right]
$$

where $\omega$ is the intrinsic frequency, $x$ is the horizontal distance, $z$ is the height, and $H$ is the scale height. Neglecting 

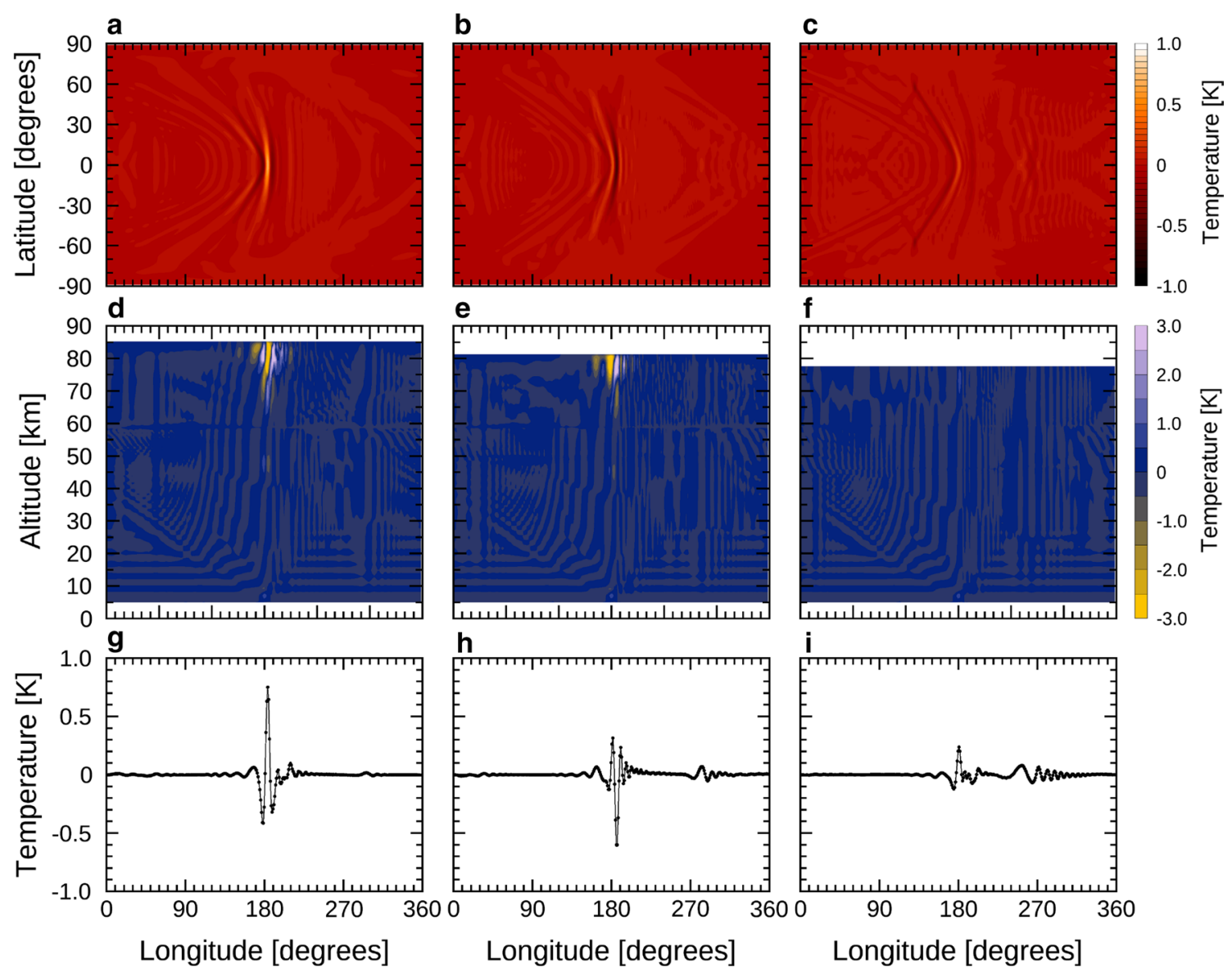

e
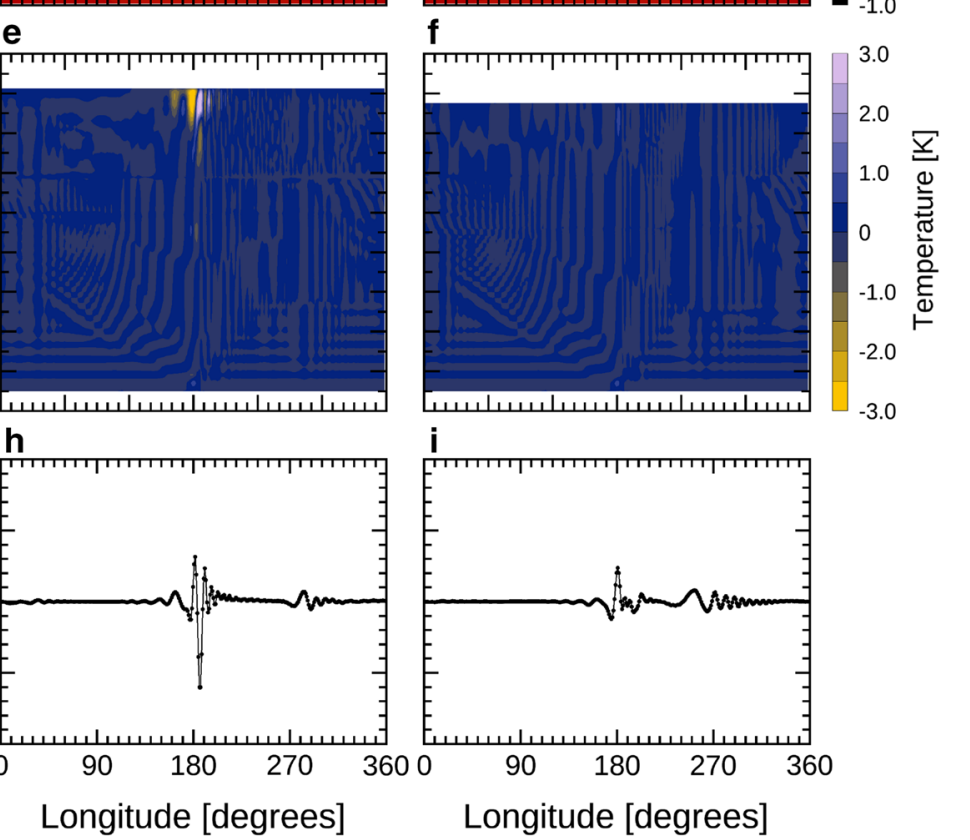

Fig. 6 Horizontal structures of the temperature disturbance, longitude-altitude cross section of the temperature disturbance on the equator, and longitudinal profiles of the temperature disturbance along the equator for the cases of the neutral layer thicknesses of $5 \mathrm{~km}(\mathbf{a}, \mathbf{d}, \mathbf{g}), 10 \mathrm{~km}(\mathbf{b}, \mathbf{e}, \mathbf{h})$, and $15 \mathrm{~km}(\mathbf{c}, \mathbf{f}, \mathbf{i})$ (see Fig. 2). Note that regions of $3 \mathrm{~K}$ and above were filled with purple, and regions of $-3 \mathrm{~K}$ and below were filled with yellow $(\mathbf{d}$, e, f)

the effect of planetary rotation, friction and thermal conduction, the dispersion relation for gravity waves propagating in the $(x, z)$ plane in an isothermal atmosphere is written as (Salby 1996):

$$
m^{2}=\frac{\omega^{2}}{c_{s}^{2}}-k^{2}+\frac{N^{2} k^{2}}{\omega^{2}}-\frac{1}{4 H^{2}}
$$

where $c_{s}^{2}$ is the sound speed. Focusing on gravity waves in a neutral layer, we set $N=0$ and assume $c_{s}^{2} \gg \omega^{2} / k^{2}$. Then, the vertical wavenumber is written as

$$
m \sim i \frac{1}{2 H} \sqrt{1+4 k^{2} H^{2}}
$$

where the sign of $m$ was chosen so that the energy density does not increase with height considering a wave generated in the lower atmosphere. By substituting Eq. (7) into Eq. (5), we obtain the following solution:

$$
w(z) \propto \exp \left[-\frac{\sqrt{1+4 k^{2} H^{2}}-1}{2 H} z\right]
$$

From this solution, the e-folding decay length $d_{e}$ is obtained as

$$
d_{e}=\frac{2 H}{\sqrt{1+4 k^{2} H^{2}}-1}
$$

The zonal wavelengths of the gravity waves reproduced in this study are larger than $1000 \mathrm{~km}$. The scale height at the altitude of $55 \mathrm{~km}$ is approximately $6.5 \mathrm{~km}$ (Seiff et al. 1985). By substituting these values into Eq. (9), we obtain $d_{e} \geq 3900 \mathrm{~km}$. This value is much larger than the thickness of the neutral layer of $<15 \mathrm{~km}$, and thus the gravity waves reach the cloud top with little attenuation in the neutral layer. This conclusion also applies to the observed stationary gravity waves, which have zonal 
wavelengths of 400-1500 km (Fukuhara et al. 2017b; Kouyama et al. 2017), yielding $d_{e}=600-8800 \mathrm{~km}$. We should note, however, that the energy density decreases with height in the neutral layer and that the difference in the neutral layer thickness can cause a difference in the wave amplitude above the neutral layer. Since the amplitude is almost constant along the altitude in the neutral layer and the energy density is proportional to the background atmospheric density and the square of the amplitude, the energy density decreases with altitude roughly in proportion to the background atmospheric density. For example, given the typical scale height of $6.5 \mathrm{~km}$ in the neutral layer, the energy transmission rate is $\exp [-(5 \mathrm{~km}) /(6.5 \mathrm{~km})]=0.463$ for a $5 \mathrm{~km}$-thick neutral layer and $\exp [-(15 \mathrm{~km}) /(6.5 \mathrm{~km})]=0.099$ for a $15 \mathrm{~km}$-thick layer; the amplitude ratio between the two cases at a common altitude above the neutral layer would be $(0.099 / 0.463)^{1 / 2} \sim 0.46$, which is comparable to the amplitude ratio shown in Fig. 6 . Note that outside of the neutral layer, because of the conservation of the energy density, the amplitude increases with height thanks to the decrease of the atmospheric density. Since a neutral layer prevents this amplitude growth, an increase of the neutral layer thickness leads to a decrease of the amplitude above the neutral layer.

Shorter wavelength waves can be attenuated in the neutral layer more effectively. For example, the amplitudes of gravity waves with horizontal wavelengths of 20 and 50 $\mathrm{km}$ would decrease by a factor of 0.29 and 0.70 , respectively, in a $5 \mathrm{~km}$-thickness neutral layer, by a factor of 0.09 and 0.50 , respectively, in a $10 \mathrm{~km}$-thickness neutral layer, and by a factor of 0.03 and 0.35 , respectively, in a 15 $\mathrm{km}$-thickness neutral layer. These attenuation rates were calculated by substituting the neutral layer thickness of $\Delta z=5,10$, and $15 \mathrm{~km}, H=6.5 \mathrm{~km}$, and $\lambda_{x}=20 \mathrm{~km}$ and $50 \mathrm{~km}$ into $\exp \left[-\left(\Delta z / d_{e}\right)\right]$. Based on these estimates, we suppose that gravity waves with horizontal wavelengths shorter than tens of kilometers are significantly attenuated in the neutral layer and hardly be observed at the cloud top. Statistics of the observed wavelength would provide information on the excitation source of the gravity waves. For instance, Piccialli et al. (2014) detected gravity waves with horizontal wavelengths of $3-21 \mathrm{~km}$ from close-up observations by Venus Monitoring Camera onboard Venus Express with an ultraviolet filter (wavelength $\sim 365 \mathrm{~nm}$ ). Though the waves seem to be relatively concentrated over Ishtar Terra, we consider they are not excited below the cloud but excited above the neutral layer because of the short horizontal wavelengths. Wave generation by convection in the cloud (Imamura et al. 2014; Lefèvre et al. 2018) is another possibility. By contrast, Peralta et al. (2017) observed gravity waves with horizontal wavelengths of 100 to $300 \mathrm{~km}$ that appeared to be fixed to the topography by VIRTIS-M onboard Venus Express with infrared wavelengths of 3.8 and $5.0 \mu \mathrm{m}$. Considering the relatively long wavelengths, the excitation sources can be mountains.

Assuming the excitation source is mountains, we can relate the horizontal wavelength to the mountain width contributing to wave excitation. Nappo (2013) suggested that the horizontal wavelength of the dominant wave is $\lambda_{x}$ $\sim 4.4 b$, where $b$ is the half-width of the Gaussian-shaped mountain. In our model, the half-width of the wave forcing is $3^{\circ}$, corresponding to $\sim 320 \mathrm{~km}$, and thus the dominant wavelength should be approximately $1400 \mathrm{~km}$. This estimate is compatible with the zonal wavelengths seen in the model of 1300 and $1900 \mathrm{~km}$. The horizontal wavelength does not change much from the forcing region to the cloud top (Fig. 5a). The observation by LIR showed the zonal wavelength of approximately $1500 \mathrm{~km}$ above Aphrodite Terra, suggesting that the horizontal scale of the topography contributing to the excitation of the observed waves is similar to that in the model. This work demonstrated the applicability of Nappo (2013) formula to the Venusian atmosphere and suggests the possibility that the horizontal scales of the mountains contributing to wave excitation can be inferred from the features appearing at the cloud top.

\section{Conclusion}

This study revealed, using three-dimensional numerical model, that gravity waves with zonal wavelengths longer than $1000 \mathrm{~km}$ excited near the surface can propagate to the cloud top and produce temperature disturbances as observed, despite the existence of a neutral layer with the thickness of up to $15 \mathrm{~km}$ in the cloud. The results strongly suggest that the dependence of the observed wave amplitude on the local time and the latitude are not attributed to the variation of the neutral layer thickness along the local time and the latitude but is caused by excitation and/or transmission processes in the lower atmosphere.

It was also shown with an analytical approach that the horizontal wavelength basically determines the e-folding decay length in the neutral layer. Specifically, gravity waves with wavelengths longer than $100 \mathrm{~km}$ can propagate through the neutral layer with little attenuation, while those with wavelengths shorter than tens of kilometers are strongly attenuated. This implies that observations of the horizontal wavelengths of gravity waves can impose restrictions on the altitude where the waves are generated. For precise evaluation of the ability of transmission through the neutral layer for various wavelengths, the effect of convective mixing should also be considered. 


\section{Acknowledgements}

The authors would like to thank all Akatsuki team members for fruitful scientific discussion.

\section{Authors' contributions}

TY performed the simulations, model development, and scientific interpretation; TI contributed to the model development and scientific interpretation. TF and MT contributed to the scientific interpretation. All authors read and approved the final manuscript.

\section{Funding}

No financial support was received for this study.

\section{Code availability}

We have opted not to make the computer code used in the numerical model available.

\section{Competing interests}

The authors declare that they have no competing interests.

\section{Author details}

${ }^{1}$ Graduate School of Science, Rikkyo University, Tokyo 171-8501, Japan. ${ }^{2}$ Graduate School of Frontier Sciences, The University of Tokyo, Chiba 277-8561, Japan.

\section{Received: 1 April 2019 Accepted: 9 November 2019}

Published online: 20 November 2019

\section{References}

Crisp D (1989) Radiative forcing of the Venus mesosphere II. Thermal fluxes, cooling rates, and radiative equilibrium temperatures. Icarus 77(2):391-413. https://doi.org/10.1016/0019-1035(89)90096-1

Durran DR (1990) Mountain waves and downslope winds. In: Blumen W (ed) Atmospheric processes over complex terrain. Meteorological monographs, vol 23. American Meteorological Society, Boston, pp 59-81

Fukuhara T, Taguchi M, Imamura T, Nakamura M, Ueno M, Suzuki M, Iwagami N, Sato M, Mitsuyama K, Hashimoto GL, Ohshima R, Kouyama T, Ando H, Futaguchi M (2011) LIR: longwave Infrared Camera onboard the Venus orbiter Akatsuki. Earth Planets Space 63:1009-1018. https://doi.org/10.5047/ eps.2011.06.019

Fukuhara T, Taguchi M, Imamura T, Hayashitani A, Yamada T, Futaguchi M, Kouyama T, Sato TM, Takamura M, Iwagami N, Nakamura M, Suzuki M, Ueno M, Hashimoto GL, Sato M, Takagi S, Yamazaki A, Yamada M, Murakami S, Yamamoto Y, Ogohara K, Ando H, Sugiyama K, Kashimura H, Ohtsuki S, Ishii N, Abe T, Satoh T, Hirose C, Hirata N (2017a) Absolute calibration of brightness temperature of the Venus disk observed by the Longwave Infrared Camera onboard Akatsuki. Earth Planets Space 69(1):141. https://doi.org/10.1186/ s40623-017-0727-y

Fukuhara T, Futaguchi M, Hashimoto GL, Horinouchi T, Imamura T, Iwagaimi N, Kouyama T, Murakami S, Nakamura M, Ogohara K, Sato M, Sato TM, Suzuki M, Taguchi M, Takagi S, Ueno M, Watanabe S, Yamada M, Yamazaki A (2017b) Large stationary gravity wave in the atmosphere of Venus. Nat Geosci 10:85-88. https://doi.org/10.1038/ngeo2873

Haltiner GJ, Williams RT (1980) Numerical prediction and dynamic meteorology, 2d edn. Wiley, Hoboken, p 477

Hinson DP, Jenkins JM (1995) Magellan radio occultation measurements of atmospheric waves on Venus. Icarus 114(2):310-327. https://doi. org/10.1006/icar.1995.1064

Horinouchi T, Kouyama T, Lee YJ, Murakami S, Ogohara K, Takagi M, Imamura T, Nakajima K, Peralta J, Yamazaki A, Yamada M, Watanabe S (2018) Mean winds at the cloud top of Venus obtained from two-wavelength UV imaging by Akatsuki. Earth Planets Space 70(1):10. https://doi.org/10.1186/s4062 3-017-0775-3

Imamura T (2006) Meridional propagation of planetary-scale waves in vertical shear: implication for the Venus atmosphere. J Atmos Sci 63(6):1623-1636. https://doi.org/10.1175/JAS3684.1

Imamura T, Hashimoto GL (2001) Microphysics of venusian clouds in rising tropical air. J Atmos Sci 58(23):3597-3612. https://doi.org/10.1175/15200469(2001)058<3597:MOVCIR>2.0.CO;2
Imamura T, Higuchi T, Maejima Y, Takagi M, Sugimoto N, Ikeda K, Ando H (2014) Inverse insolation dependence of Venus' cloud-level convection. Icarus 228:181-188. https://doi.org/10.1016/j.icarus.2013.10.012

Imamura T, Ando H, Tellmann S, Pätzold M, Häusler B, Yamazaki A, Sato TM, Noguchi K, Futaana Y, Oschlisniok J, Limaye S, Choudhary RK, Murata Y, Takeuchi H, Hirose C, Ichikawa T, Toda T, Tomiki A, Abe T, Yamamoto Z, Noda H, Iwata T, Murakami S, Satoh T, Fukuhara T, Ogohara K, Sugiyama K, Kashimura H, Ohtsuki S, Takagi S, Yamamoto Y, Hirata N, Hashimoto GL, Yamada M, Suzuki M, Ishii N, Hayashiyama T, Lee YJ, Nakamura M (2017) Initial performance of the radio occultation experiment in the Venus orbiter mission Akatsuki. Earth Planets Space 69:137-147. https://doi.org/10.1186/s40623-017-0722-3

Kouyama T, Imamura T, Taguchi M, Fukuhara T, Sato TM, Yamazaki A, Futaguchi M, Murakami S, Hashimoto GL, Ueno M, Iwagami N, Takagi S, Takagi M, Ogohara K, Kashimura H, Horinouchi T, Sato N, Yamada M, Yamamoto Y, Ohtsuki S, Sugiyama K, Ando H, Takamura M, Yamada T, Satoh T, Nakamura M (2017) Topographical and local time dependence of large stationary gravity waves observed at the cloud top of Venus. Geophys Res Lett 44:12098-12105. https://doi.org/10.1002/2017GL075792

Lefèvre M, Lebonnois S, Spiga A (2018) Three-dimensional turbulence-resolving modeling of the Venusian cloud layer and induced gravity waves: inclusion of complete radiative transfer and wind shear. J Geophys Res Planets 123(10):2773-2789. https://doi.org/10.1029/2018JE005679

Nappo CJ (2013) An Introduction to atmospheric gravity waves. International geophysics, vol 102. Academic press, Cambridge, p 359

Navarro T, Schubert G, Lebonnois S (2018) Atmospheric mountain wave generation on Venus and its influence on the solid planet's rotation rate. Nat Geosci 11(7):487-491. https://doi.org/10.1038/s41561-018-0157-x

Peralta J, Hueso R, Sánchez-Lavega A, Lee YJ, Muñoz AG, Kouyama T, Sagawa H, Sato TM, Piccioni G, Tellmann S, Imamura T, Satoh T (2017) Stationary waves and slowly moving features in the night upper clouds of Venus. Nature Astron. https://doi.org/10.1038/s41550-017-0187

Piccialli A, Titov DV, Sanchez-Lavega A, Peralta J, Shalygina O, Markiewicz WJ, Svedhem H (2014) High latitude gravity waves at the Venus cloud tops as observed by the Venus Monitoring Camera on board Venus Express. Icarus 227:94-111. https://doi.org/10.1016/j.icarus.2013.09.012

Rossow WB, Genio ADD, Eichler T (1990) Cloud-tracked winds from pioneer Venus OCPP images. J Atmos Sci 47(17):2053-2084

Salby ML (1996) Chapter 14 Atmospheric waves. In: Fundamentals of atmospheric physics. International geophysics, vol 61. Academic Press, Cambridge, pp 426-485. https://doi.org/10.1016/S0074-6142(96)80051-9

Schubert G, Walterscheid RL (1984) Propagation of small-scale acoustic-gravity waves in the Venus atmosphere. J Atmos Sci 41(7):1202-1213

Seiff A, Schofield JT, Kliore AJ, Taylor FW, Limaye SS, Revercomb HE, Sromovsky LA, Kerzhanovich W, Moroz VI, Marov MY (1985) Models of the structure of the atmosphere of Venus from the surface to 100 kilometers altitude. Adv Space Res 5(11):3-58. https://doi.org/10.1016/0273-1177(85)90197-8

Taguchi M, Fukuhara T, Imamura T, Nakamura M, Iwagami N, Ueno M, Suzuki M, Hashimoto GL, Mitsuyama K (2007) Longwave Infrared Camera onboard the Venus Climate Orbiter. Adv Space Res 40:861-868. https://doi.org/10.1016/j. asr.2007.05.085

Tellmann S, Pätzold M, Häusler B, Bird MK, Tyler GL (2009) Structure of the Venus neutral atmosphere as observed by the Radio Science experiment VeRa on Venus Express. J Geophys Res. https://doi.org/10.1029/2008JE003204

Woo R, Ishimaru A (1981) Eddy diffusion coefficient for the atmosphere of Venus from radio scintillation measurements. Nature 289:383-384. https://doi. org/10.1038/289383a0

Woo R, Armstrong JW, Kliore AJ (1982) Small-scale turbulence in the atmosphere of Venus. Icarus 52(2):335-345. https://doi.org/10.1016/0019-1035(82)90116 $-6$

Young RE, Walterscheid RL, Schubert G, Seiff A, Linkin VM, Lipatov AN (1987) Characteristics of gravity waves generated by surface topography on Venus: comparison with the Vega balloon results. J Atmos Sci 44(18):2628-2639

Young RE, Walterscheid RL, Schubert G, Pfister L, Houben H, Bindschadler DL (1994) Characteristics of finite amplitude stationary gravity waves in the atmosphere of Venus. J Atmos Sci 51(13):1857-1875 